\title{
Improved Flux Pinning Performance of GdBCO Superconductor Bulks by $\mathrm{Gd}_{3} \mathrm{ZrO}_{7}$ Additions
}

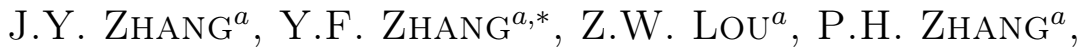 \\ C.Y. $\mathrm{LI}^{a}$, J.W. YUAN ${ }^{a}$, X.J. ZHANG ${ }^{a}, \mathrm{~L} \mathrm{PENG}^{a}$, \\ Y.X. MA ${ }^{a}$, J.M. XU ${ }^{b}$, G.T. YANG ${ }^{b}$ AND M. IZUMI ${ }^{c}$ \\ ${ }^{a}$ College of Mathematics and Physics, Shanghai University of Electric Power, \\ 1851 Hucheng Ring Road, New Pudong District, Shanghai 201306, People's Republic of China \\ ${ }^{b}$ State Key Laboratory of Space Power Technology, \\ Shanghai Institute of Space Power-Sources, Shanghai 200245, China \\ ${ }^{c}$ Laboratory of Applied Physics, Tokyo University of Marine Science and Technology, \\ 2-1-6 Etchujima, Koto-ku, Tokyo 135-8533, Japan
}

Received: 22.01.2021 \& Accepted: 06.06.2021

Doi: $10.12693 /$ APhysPolA.140.40

*e-mail: $\quad 20090000180$ shiep.edu.cn

\begin{abstract}
Four $\mathrm{GdBa}_{2} \mathrm{Cu}_{3} \mathrm{O}_{7-\delta}\left(\mathrm{GdBCO}\right.$ or $\left.\mathrm{Gd}_{123}\right)$ superconductor bulks doped with different amounts of $\mathrm{Gd}_{3} \mathrm{ZrO}_{7}$ particles have been successfully prepared by the modified top-seeded melt-texture growth. Superconducting properties and microstructures of these bulks have been investigated in detail. All GdBCO bulks exhibit superior superconductivity with the onset critical temperature $\left(T_{\mathrm{c} \text { onset }}\right)$ above $94 \mathrm{~K}$, and the highest critical current density $\left(J_{\mathrm{c}}\right)$ of $49.1 \mathrm{kA} / \mathrm{cm}^{2}$ was obtained in the GdBCO bulk doped with 0.8 mol. $\% \mathrm{Gd}_{3} \mathrm{ZrO}_{7}$ particles at $77 \mathrm{~K}$ in self-field. During the top-seeded melt-texture growth process, the $\mathrm{Gd}_{3} \mathrm{ZrO}_{7}$ additions can react with the $\mathrm{GdBCO}$ phase, forming the $\mathrm{BaZrO}_{3}$ particles and intensifying the $\mathrm{Gd} / \mathrm{Ba}$ substitution, which can improve the flux pinning performance of the GdBCO bulk. As a result, $J_{\mathrm{c}}$ was enhanced and the trapped flux density significantly increased with the increase of $\mathrm{Gd}_{3} \mathrm{ZrO}_{7}$ additions. The maximum trapped flux density of $0.56 \mathrm{~T}$ obtained in the GdBCO bulk with $0.8 \mathrm{~mol} . \% \mathrm{Gd}_{3} \mathrm{ZrO}_{7}$ additions is more than three times as high as that of the undoped bulk. The squared stripes around the seed crystal were found in the doped bulks, and were identified as the macroscopic appearance of inhomogeneous microstructure in which the $\mathrm{Gd}_{2} \mathrm{BaCuO}_{5}(\mathrm{Gd} 211)$ free region and the Gd211-containing region were separated by a $\mathrm{BaZrO}_{3}$-and-Ag layer in a small region. But such microstructural inhomogeneity can be well improved with the increase of $\mathrm{Gd}_{3} \mathrm{ZrO}_{7}$ additions. All these results show that $\mathrm{Gd}_{3} \mathrm{ZrO}_{7}$ additions improve the pinning performance of $\mathrm{GdBCO}$ superconductor bulks.
\end{abstract}

topics: GdBCO superconductor bulk, top-seeded melt-texture growth, $\mathrm{Gd}_{3} \mathrm{ZrO}_{7}$ additions, critical current density

\section{Introduction}

Because of higher critical current density $\left(J_{\mathrm{c}}\right)$ and the ability to trap higher magnetic field compared with the low temperature superconductors, $\mathrm{REBa}_{2} \mathrm{Cu}_{3} \mathrm{O}_{7-\delta}$ (REBCO or RE123, $\mathrm{RE}=$ rare earth elements, such as $\mathrm{Y}, \mathrm{Gd}, \mathrm{Sm}, \mathrm{Nd}$, etc.) high temperature superconductors have attracted widespread attention and been widely used in various practical applications, such as magnetic levitation, motors, flywheel energy storage systems, etc. [1-5]. However, the improvement of superconducting property is greatly restricted due to the existence of weak links between the superconducting grains, the poor magnetic flux pinning ability, etc. The top-seeded melt-texture growth (TSMG) method has been widely used to prepare REBCO superconductor bulks with excellent properties, but the shrinkage of REBCO bulks due to the diffusion and loss of liquid phase during crystal growth can degrade the superconductivity of REBCO bulks and even lead to the failure of sample preparation. Meanwhile, the top-seeded infiltration and growth (TSIG) allows to avoid such problems and to have a negligible shrinkage of the bulks since the introduction of a liquid phase source can provide the sufficient liquid phase to the precursor pellet [6-9]. Consequently, in this study, we prepare the GdBCO superconductor bulks by the TSMG method and introduce the Y123 pellet as the liquid phase source in order to prepare high-performance GdBCO superconductor bulks with negligible shrinkage. 
It has been proved that $J_{\mathrm{c}}$ and trapped magnetic flux of the REBCO superconductor bulk can be improved by introducing appropriate second phase particles as effective pinning centers [10-12] while Zr-containing particles can be used as effective doping particles to promote the improvement of REBCO superconductors [13-15] performance. In addition, considering that the second peak effect caused by the $\mathrm{Gd} / \mathrm{Ba}$ substitution can well improve

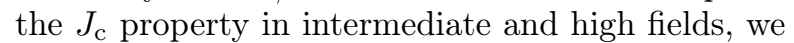
chose the $\mathrm{Gd}_{3} \mathrm{ZrO}_{7}$ particles as the doping particles. Generally, the optimal amount of the doping particles is around $0.4 \mathrm{~mol} \%[14,16-19]$, thus the doping amount of the $\mathrm{Gd}_{3} \mathrm{ZrO}_{7}$ particles was set in the range of $0.0-0.8 \mathrm{~mol} \%$ in this study.

Four GdBCO superconductor bulks doped with 0.0-0.8 mol.\% $\mathrm{Gd}_{3} \mathrm{ZrO}_{7}$ particles have been successfully fabricated by the modified TSMG method. We systematically studied the influence of different doping amounts of $\mathrm{Gd}_{3} \mathrm{ZrO}_{7}$ particles on the critical temperature, the critical current density, the trapped flux density and the microstructure of these GdBCO superconductor bulks.

\section{Experimental}

\subsection{Preparation of $\mathrm{Gd}_{3} \mathrm{ZrO}_{7}$ powders}

The $\mathrm{Gd}_{3} \mathrm{ZrO}_{7}$ powders have been prepared by solid state reaction. Commercial $\mathrm{Gd}_{2} \mathrm{O}_{3}(99.9 \%)$ and $\mathrm{ZrO}_{2}(99.9 \%)$ powders were mixed in the molar ratio of $\mathrm{Gd}: \mathrm{Zr}=3: 1$ and ground in a mortar for $3 \mathrm{~h}$. We put the well mixed powders into the resistance furnace for sintering, with sintering temperature up to $940^{\circ}$ in $5 \mathrm{~h}$, and keeping for $10 \mathrm{~h}$. Then, we ground the sintered powders again for $3 \mathrm{~h}$ and sintered again as before. After three times of sintering and four times of grinding, the $\mathrm{Gd}_{3} \mathrm{ZrO}_{7}$ powders were prepared. The XRD analysis was applied to identify the composition of powders, and the XRD diffraction patterns of powders are given in Fig. 1. It was found that the main peak of the XRD diffraction patterns is obvious, which confirms the successful preparation of the $\mathrm{Gd}_{3} \mathrm{ZrO}_{7}$ powders with high purity.

\subsection{Preparation of GdBCO superconductor bulks}

The $\mathrm{Gd}_{3} \mathrm{ZrO}_{7}$ powders and commercially pure powders of $\mathrm{Gd} 123$ (99.9\%) and $\mathrm{Gd}_{2} \mathrm{BaCuO}_{5}$ (Gd211) (99.9\%) were used as a starting material in an initial composition of Gd123 +40 mol.\% $\mathrm{Gd} 211+x$ mol.\% $\mathrm{Gd}_{3} \mathrm{ZrO}_{7}(x=0,0.2,0.4,0.8)$, which are denoted as S0, S2, S4 and S8, respectively. In order to improve the mechanical property and inhibit the coarsening of Gd211 particles, $10 \mathrm{wt} \% \mathrm{Ag}_{2} \mathrm{O}$ and $0.5 \mathrm{wt} \% \mathrm{Pt}$ were also added. The precursor powders were mixed thoroughly in a ball milling machine and then pressed into a cylinder pellet with the diameter of $25 \mathrm{~mm}$ and the thickness of $12 \mathrm{~mm}$ as the precursor pellet. Commercially

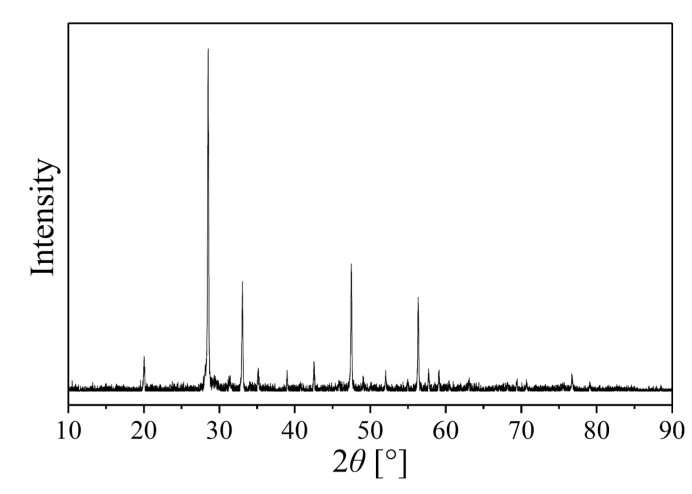

Fig. 1. XRD diffraction patterns of $\mathrm{Gd}_{3} \mathrm{ZrO}_{7}$ powders prepared by solid state reaction.

pure Y123 powders were also pressed into a cylinder pellet with the diameter of $25 \mathrm{~mm}$ and the thickness of $3 \mathrm{~mm}$ as the liquid phase source pellet. The Y123 pellet was put under the GdBCO precursor to provide sufficient liquid phase to the precursor pellet. A NdBCO seed crystal, whose size is $2 \times 2 \times 0.5 \mathrm{~mm}^{3}$, was placed at the center of the top surface of the precursor pellet. The $\mathrm{Y}_{2} \mathrm{O}_{3}$ pellet was put under the Y123 pellet to avoid the reaction between $\mathrm{Al}_{2} \mathrm{O}_{3}$ substrate and liquid phase source. The entire arrangement was put into a box furnace for the TSMG process. The temperature profile is as follows. The maximum temperature $\left(T_{\max }\right)$ was set at $1079^{\circ}$ and kept for $1 \mathrm{~h}$, then it was reduced to $1008^{\circ}$, followed by cooling at a rate of $0.3^{\circ} / \mathrm{h}$ for $30^{\circ}$ and finally cooled to room temperature. Subsequently, the annealing process was carried out in the high purity oxygen flow to transform the structure from the tetragonal non-superconducting phase to the orthogonal superconducting phase. The sintered samples were firstly heated to $450^{\circ}$ in $5 \mathrm{~h}$ and kept for $40 \mathrm{~h}$, followed by slowly cooling to $350^{\circ}$ in $140 \mathrm{~h}$, and then the temperature was decreased to $300^{\circ}$ in $30 \mathrm{~h}$. Finally, it was cooled down to room temperature. The details of the preparation process are described in $[13,19]$.

\subsection{Measurement of characteristics}

For the measurement of trapped flux density, the bulks were cooled down to the liquid nitrogen temperature under a magnetic field of $1 \mathrm{~T}$. After 30 minutes, the applied field was removed and the distribution of the trapped flux density could be obtained by the Hall probe sensor with the distance of $0.5 \mathrm{~mm}$ between the probe and the sample surface. In order to study the effect of $\mathrm{Gd}_{3} \mathrm{ZrO}_{7}$ additions on the superconducting property of the GdBCO superconductor bulk, small rectangular specimens with the size of $2 \times 2 \times 1 \mathrm{~mm}^{3}$ were cut from different positions of these superconductor bulks for the measurement of their superconducting properties. Two specimens were cut under the seed, labeled as $\mathrm{C} 1$ and $\mathrm{C} 2$ based on the distance from the top surface to the bottom, and two other specimens were cut 


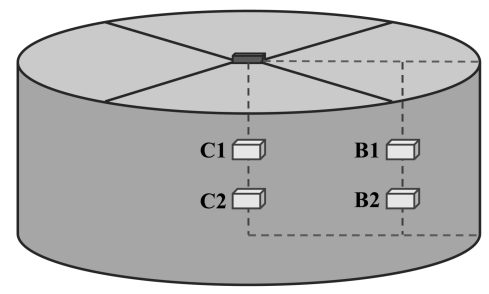

Fig. 2. Schematic diagram of the positions of four specimens cut from the GdBCO superconductor bulk.

near the boundary, labeled as B1 and B2 based on the distance from the top surface to the bottom. The specific positions of these specimens have been given in Fig. 2.

The DC magnetization measurements were carried out by using the physical property measurement system (PPMS), and the $J_{\mathrm{c}}$ values were deduced based on the extended Bean critical state model [20]. The microstructures of these bulks were observed through the scanning electron microscope (SEM), while the elemental distribution was analyzed by the energy-dispersive X-ray spectroscopy (EDX).

\section{Results and discussion}

Four single domain GdBCO superconductor bulks with different doping amounts of $\mathrm{Gd}_{3} \mathrm{ZrO}_{7}$ particles have been successfully fabricated by the modified TSMG method, as shown in Fig. 3. It can be seen that the $\mathrm{NdBCO}$ seed crystals of all bulks are kept intact and the fourfold growth sector can be clearly observed, which means good single domain performances of these four GdBCO superconductor bulks. An interesting phenomenon is observed in which the bulks doped with the $\mathrm{Gd}_{3} \mathrm{ZrO}_{7}$ particles show squared stripes around the seed crystal, in contrast to undoped bulk where this phenomenon does not occur. So we infer that the squared stripes are caused by the $\mathrm{Gd}_{3} \mathrm{ZrO}_{7}$ particles. In some studies on the REBCO superconductor bulks doped with $\mathrm{ZrO}_{2}$ powders [18-21], inhomogeneous microstructures were proposed, such as the second phase free band-the second phase rich band-the second phase diluted band and a growth cycle consisting of a RE211 free layer-porous narrow layer-RE211 high concentration layer. In [22], the structure that the squared narrow bands formed by $\mathrm{BaZrO}_{3}$ and Y211 particles enclosing the YBCO single domain regions free of Y211 particles was considered in the YBCO bulk with $\mathrm{BaZrO}_{3}$ additions. According to these studies, it is reasonable to deduce that the squared stripes around the seed crystal shown in the doped samples are the macroscopic appearances of inhomogeneous microstructure. Moreover, it can be found that the region where the squared stripes are distributed becomes smaller with the increase of $\mathrm{Gd}_{3} \mathrm{ZrO}_{7}$ additions.

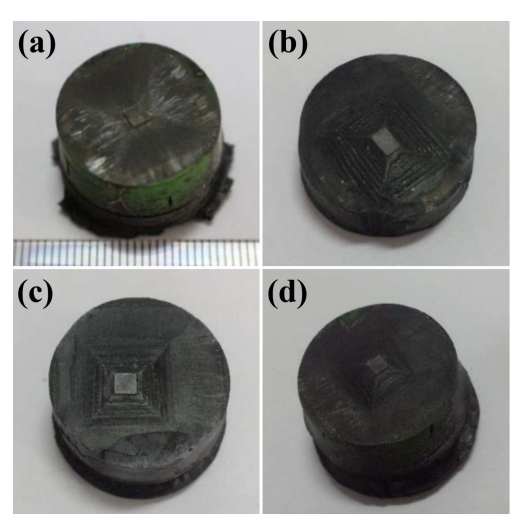

Fig. 3. Top view of GdBCO superconductor bulks with different doping amounts of $\mathrm{Gd}_{3} \mathrm{ZrO}_{7}$ particles: (a) S0, (b) S2, (c) S4, (d) S8.

The temperature dependence of magnetization of specimens cut from different positions, C1, C2, B1 and $\mathrm{B} 2$, of the GdBCO superconductor bulks with different amounts of $\mathrm{Gd}_{3} \mathrm{ZrO}_{7}$ particles are shown in Fig. 4. The case of the position dependence of $T_{c \text {, onset }}$ of specimens cut from four GdBCO superconductor bulks with different amount of $\mathrm{Gd}_{3} \mathrm{ZrO}_{7}$ particles was presented in Fig. 4e. It can be clearly seen that except for B1 position, the $T_{c \text {, onset val- }}$ ues of some doped bulks are slightly lower than that of the undoped bulk. However, the addition of $\mathrm{Gd}_{3} \mathrm{ZrO}_{7}$ particles does not degrade the superconductivity of GdBCO bulk because all specimens

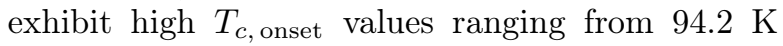
to $95.9 \mathrm{~K}$.

Figure 5 shows the magnetic field $\mu H$ dependence of critical current density $J_{\mathrm{c}}$ of specimens, cut from different positions ( $\mathrm{C} 1, \mathrm{C} 2, \mathrm{~B} 1$ and $\mathrm{B} 2)$ of the GdBCO superconductor bulks with different amounts of $\mathrm{Gd}_{3} \mathrm{ZrO}_{7}$ particles at $77 \mathrm{~K}$. Plots of $J_{\mathrm{c}}$ in self-field as a function of the amount of $\mathrm{Gd}_{3} \mathrm{ZrO}_{7}$ additions are shown in Fig. 6, in order to present the occurrence of these bulks in low fields. The $J_{\mathrm{c}}$ values of specimens cut from different positions of a superconductor bulk are usually different, and there are various causes of that, e.g., the existence of a seed crystal, the inhomogeneous distribution of the second phase particles, etc. $[6,23,24]$. Based on the results shown in Figs. 5 and 6, we can also find that there are larger or smaller differences between the four specimens for both undoped and doped bulks, however these differences decrease in the doped bulks as compared with the undoped bulk. This indicates that the addition of the $\mathrm{Gd}_{3} \mathrm{ZrO}_{7}$ particles has a position effect on the microstructural uniformity.

It can be seen in Fig. 6 that the self-field $J_{\mathrm{c}}$ of the specimens under the seed (C1 and C2) increases, however such increase is not obvious in the GdBCO bulk doped with 0.8 mol. $\% \mathrm{Gd}_{3} \mathrm{ZrO}_{7}$ particles, where a significant decrease of $J_{\mathrm{c}}$ is observed at $\mathrm{C} 1$ position. For the specimens near the boundary (B1 and B2), the self-field $J_{\mathrm{c}}$ is degraded by the 

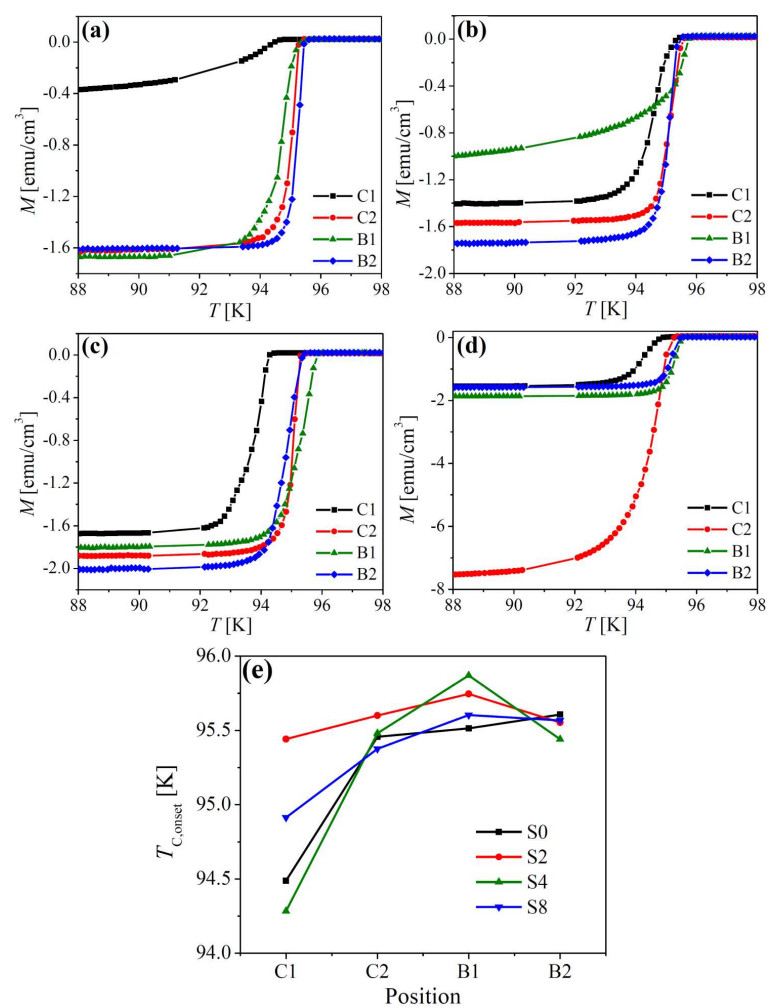

Fig. 4. Temperature $(T)$ dependence of magnetization $(M)$ of specimens cut from different positions, C1 (squares), C2 (circles), B1 (triangles), and B2 (diamonds), of the GdBCO superconductor bulks with different amounts of $\mathrm{Gd}_{3} \mathrm{ZrO}_{7}$ particles: (a) S0, (b) S2, (c) S4, (d) S8. (e) Position dependence of $T_{\mathrm{c} \text {,onset }}$ of specimens cut from four GdBCO superconductor bulks with different amounts of $\mathrm{Gd}_{3} \mathrm{ZrO}_{7}$ particles: S0, S2, $\mathrm{S} 4$, and S8.
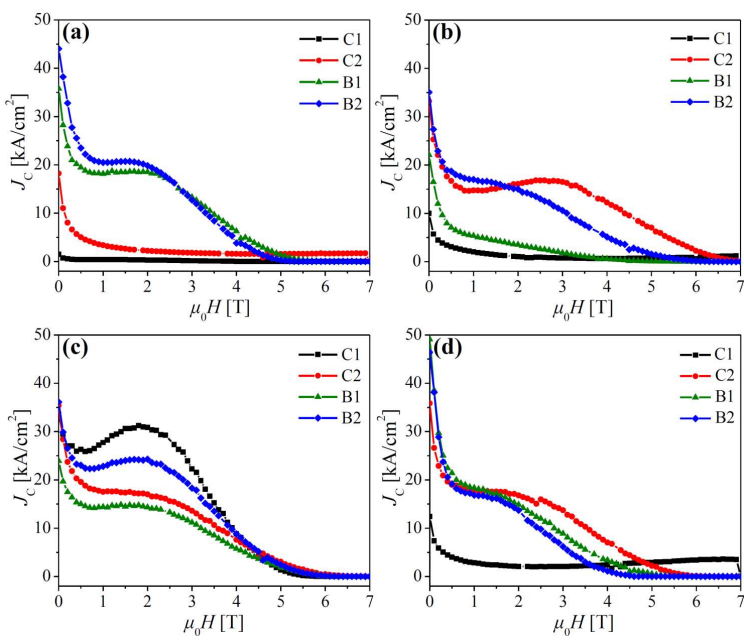

Fig. 5. Magnetic field $(\mu H)$ dependence of critical current density $\left(J_{\mathrm{c}}\right)$ of specimens cut from different positions, C1 (squares), C2 (circles), B1 (triangles) and B2 (diamonds) of the GdBCO superconductor bulks with different amount of $\mathrm{Gd}_{3} \mathrm{ZrO}_{7}$ particles: (a) S0, (b) S2, (c) S4, (d) S8 at $77 \mathrm{~K}$.

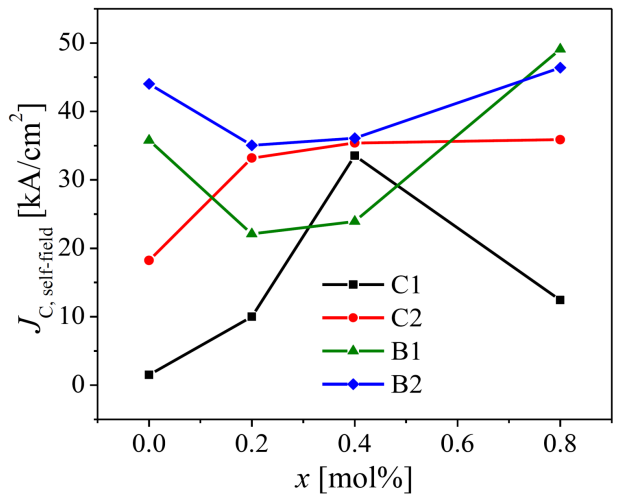

Fig. 6. Plots of $J_{\mathrm{c}}$ in self-field $\left(J_{\mathrm{c}, \text { self-field }}\right)$ of specimens from different positions, C1 (squares), $\mathrm{C} 2$ (circles), B1 (triangles) and B2 (diamonds) as a function of the amount of $\mathrm{Gd}_{3} \mathrm{ZrO}_{7}$ additions $(x)$.

$\mathrm{Gd}_{3} \mathrm{ZrO}_{7}$ additions first, and then improved with the increase of the $\mathrm{Gd}_{3} \mathrm{ZrO}_{7}$ additions. The maximum self-field $J_{\mathrm{c}}=49.1 \mathrm{kA} / \mathrm{cm}^{2}$ at $77 \mathrm{~K}$ is obtained in the $\mathrm{B} 1$ of the GdBCO bulk doped with $0.8 \mathrm{~mol} . \%$ $\mathrm{Gd}_{3} \mathrm{ZrO}_{7}$ particles. In addition, it can be observed that the second peak effect becomes more obvious with the addition of $\mathrm{Gd}_{3} \mathrm{ZrO}_{7}$ particles, but less obvious in the GdBCO bulk doped with 0.8 mol.\% $\mathrm{Gd}_{3} \mathrm{ZrO}_{7}$ particles.

For the undoped bulk (S0), the $J_{\mathrm{c}}$ values of specimens near the boundary are higher than those of specimens under the seed $(\mathrm{B} 1>\mathrm{C} 1$ and $\mathrm{B} 2>\mathrm{C} 2)$ in low fields. It is a typical phenomenon of REBCO superconductor bulks [25]. During the growth of REBCO single domain superconductor bulks, smallsized Gd211 particles are pushed to the growth front due to the pushing/trapping theory $[21,25,26]$ and serve as pinning centers greatly improving their $J_{\text {c }}$ properties. This, in turn, results in higher $J_{\mathrm{c}}$ of specimens near the boundary than that of the specimens under the seed in low fields. The slight second peaks are shown in B1 and B2 of the undoped bulk, which are usually caused by the $\mathrm{Gd} / \mathrm{Ba}$ substitution.

One can observe in Figs. 5 and 6 that when a small amount of $\mathrm{Gd}_{3} \mathrm{ZrO}_{7}$ particles was introduced into the GdBCO superconductor bulks (S2), the self-field $J_{\mathrm{c}}$ values of the specimens under the seed increased, while the self-field $J_{\mathrm{c}}$ values of the specimens near the boundary decreased. It can be inferred that the small doping amount of the $\mathrm{Gd}_{3} \mathrm{ZrO}_{7}$ particles cannot well improve the $J_{\mathrm{c}}$ property of the whole GdBCO bulk in low fields. In intermediate and high fields, however, the $J_{\mathrm{c}}$ properties were well improved, especially for $\mathrm{C} 2$ of $\mathrm{S} 2$, exhibiting a clear second peak in the $J_{\mathrm{c}}(H)$ dependence. In the case of the GdBCO bulk doped with $0.4 \mathrm{~mol} . \% \mathrm{Gd}_{3} \mathrm{ZrO}_{7}$ particles (S4), the $J_{\mathrm{c}}$ property in low fields was slightly improved, while a significant improvement of $J_{\mathrm{c}}$ for almost all specimens was observed in intermediate and high fields where a clear second peak appears. Although S8 do not 


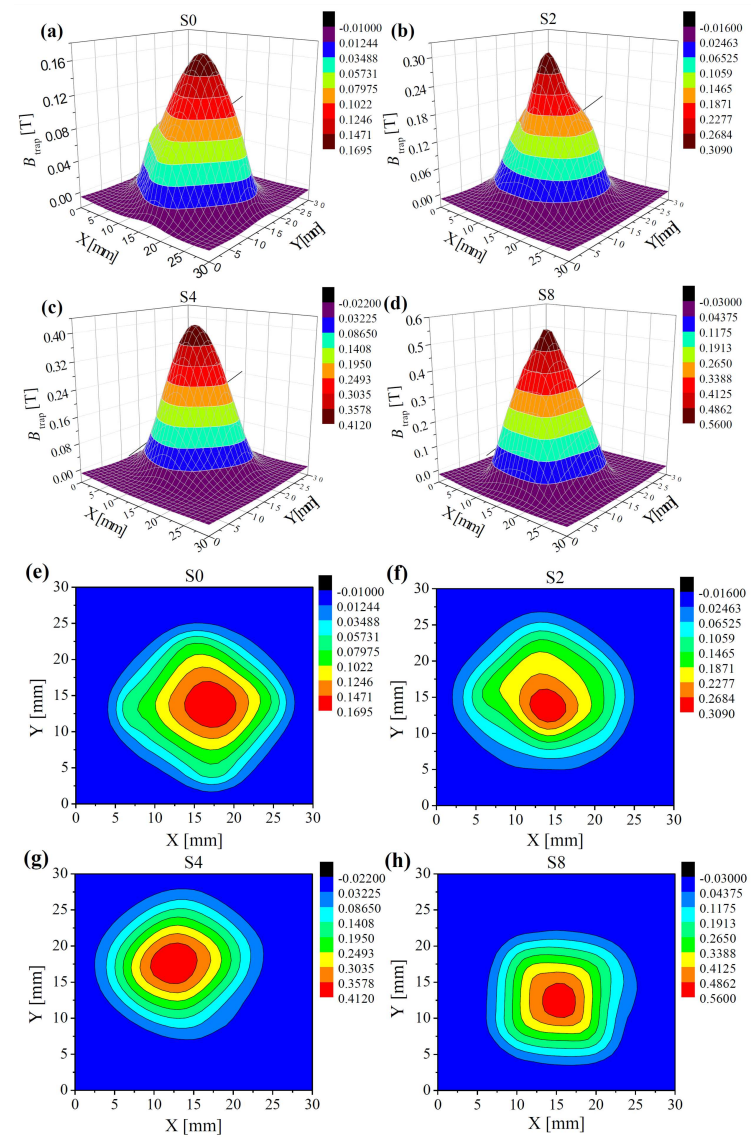

Fig. 7. Trapped flux density $\left(B_{\text {trap }}\right)$ of GdBCO superconductor bulks with different doping amounts of $\mathrm{Gd}_{3} \mathrm{ZrO}_{7}$ particles: (a) S0, (b) S2, (c) S4, (d) S8; parts (e), (f), (g), and (h) are the top view of (a), (b), (c), and (d), respectively.

exhibit the secondary peak, the self-field $J_{\mathrm{c}}$ of S8 was significantly improved, especially for the specimens near the boundary. Therefore, we infer that the addition of $\mathrm{Gd}_{3} \mathrm{ZrO}_{7}$ particles has a relevant impact on the improvement of $J_{\mathrm{c}}$ property in the whole field range, and the $\mathrm{Gd}_{3} \mathrm{ZrO}_{7}$ additions may intensify the $\mathrm{Gd} / \mathrm{Ba}$ substitution.

Figure 7 shows the trapped flux density $\left(B_{\text {trap }}\right)$ of GdBCO superconductor bulks with different doping amounts of $\mathrm{Gd}_{3} \mathrm{ZrO}_{7}$ particles. It is clear that the trapped field profiles of all samples exhibit good symmetry and conical shape, meaning that both undoped and doped GdBCO bulks exhibit superior single domain properties $[1,14,27,30]$. It can be found, in addition, that the trapped field of GdBCO bulks continuously increase with the doping amount of $\mathrm{Gd}_{3} \mathrm{ZrO}_{7}$ particles. The maximum trapped flux density value of $0.56 \mathrm{~T}$ is obtained in the bulk doped with 0.8 mol. $\% \mathrm{Gd}_{3} \mathrm{ZrO}_{7}$ particles. Note that it is more than three times as high as that of the undoped bulk whose maximum trapped flux density value is $0.17 \mathrm{~T}$. The trapped flux density $B_{\text {trap }}$ is proportional to the grain size and $J_{\mathrm{c}}$, according to the equation $B_{\text {trap }}=A \mu J_{\mathrm{c}} R$, where $A$ is the constant, $\mu$ is the permeability of vacuum, $J_{\mathrm{c}}$ is the

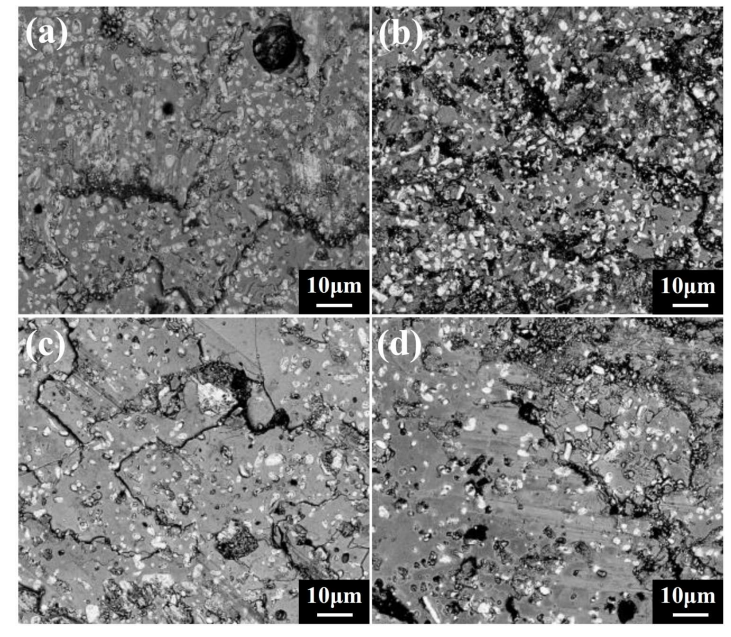

Fig. 8. SEM micrographs (at a magnification of $3000 \times)$ of B1 specimens of the GdBCO superconductor bulks with different doping amounts of $\mathrm{Gd}_{3} \mathrm{ZrO}_{7}$ particles: (a) S0, (b) S2, (c) S4, (d) S8.

critical current density and $R$ is the radius of the grain $[1,31,32]$. Since there is little difference in sizes between these bulks, we can conclude that the enhancement of $B_{\text {trap }}$ is mainly due to the increase of $J_{\mathrm{c}}$ with the increase of the $\mathrm{Gd}_{3} \mathrm{ZrO}_{7}$ additions. Therefore, it further proves that $\mathrm{Gd}_{3} \mathrm{ZrO}_{7}$ additions play a crucial role in improving the $J_{\mathrm{c}}$ property, thus enhancing the trapped flux density of the GdBCO superconductor bulk.

A high-performance superconductor must have an ideal microstructure, and the size and distribution of the second phase particles are important for its superior superconductivity. In general, the Gd211 particles with the appropriate size can be used as the pinning centers, increasing the $J_{\mathrm{c}}$ values of the superconductor bulks $[26,28,33]$. The SEM micrographs of B1 specimens of the GdBCO superconductor bulks with different doping amount of $\mathrm{Gd}_{3} \mathrm{ZrO}_{7}$ particles are presented in Fig. 8, from which the distribution of the Gd211 particles can be observed. The white particles, which are identified as the Gd211 particles, are distributed in the four samples. Obviously, the content of the Gd211 particles distributed in the $\mathrm{B} 1$ specimen of the GdBCO bulk continuously decreases with the increase of $\mathrm{Gd}_{3} \mathrm{ZrO}_{7}$ additions. The causes of this phenomenon will be systematically analyzed later.

In order to further analyze the Gd211 particles distributed in these GdBCO bulks, the size of the Gd211 particles shown in Fig. 8 was counted by the software of Nano measurer 1.2, as shown in Fig. 9. The horizontal axis shows the size range, while the vertical axis shows the number of the Gd211 particles within a certain size range as a percentage of the total number of the Gd211 particles counted in each sample. The curves are obtained by fitting the size distribution of the Gd211 particles and are represented by the red solid curves in Fig. 9, thus the size distribution and the average size of the Gd211 


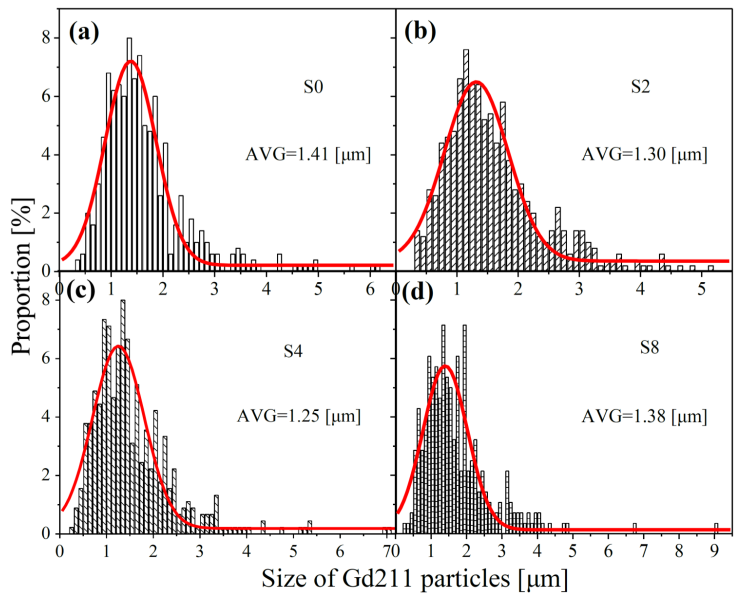

Fig. 9. The size distribution of the Gd211 particles in the GdBCO superconductor bulks with different doping amounts of $\mathrm{Gd}_{3} \mathrm{ZrO}_{7}$ particles: (a) S0, (b) $\mathrm{S} 2$, (c) S4, (d) S8. The red solid curves represent the fitted curves obtained by fitting the size distribution of the Gd211 particles. The average size values of the Gd211 particles (AVG) obtained from the peak position of each fitted curve are also given.

particles in each bulk can be predicted. The average size values of the Gd211 particles (AVG) are obtained from the peak position of each fitted curve. The average size of the Gd211 particles decreases with the increase of $\mathrm{Gd}_{3} \mathrm{ZrO}_{7}$ additions when the doping amount is less than $0.8 \mathrm{~mol} \%$, while the average size of the Gd211 particles in S8 increase compared with that in other doped bulks (S2 and S4). However, when compared with the undoped bulk, the average size of the Gd211 particles in all doped bulks decreases. We can infer, therefore, that the $\mathrm{Gd}_{3} \mathrm{ZrO}_{7}$ additions have a significant impact on the refinement of the Gd211 particles, but the excessive $\mathrm{Gd}_{3} \mathrm{ZrO}_{7}$ additions can make the Gd211 particles coarser as compared with the GdBCO bulk with a low doping amount.

Figure 10 exhibits the SEM diagrams at a higher magnification $(5000 \times)$ of specimens at B1 position of the GdBCO superconductor bulks with different doping amounts of $\mathrm{Gd}_{3} \mathrm{ZrO}_{7}$ particles. The stoichiometry of various spots labeled in Fig. 10 were identified by EDS analysis and the results are listed in Table I. It can be seen that all doped bulks contain not only Gd211 particles, but also $\mathrm{BaZrO}_{3}$ particles and liquid phase compound $\left(\mathrm{BaCuO}_{2}+\mathrm{CuO}\right)$, as marked in Fig. 10 (spots $\mathrm{E}$ and $\mathrm{F}$ of S2, spots $\mathrm{J}, \mathrm{K}$ and $\mathrm{L}$ of $\mathrm{S} 4$, as well as spots $\mathrm{P}$ and $\mathrm{Q}$ of $\mathrm{S} 8$ ). In many studies on REBCO superconductors doped with Zr-containing particles, the Zrcontaining particles tend to react with RE123 and form $\mathrm{BaZrO}_{3}$ particles [33-35]. Therefore, we infer that the reaction between $\mathrm{Gd}_{3} \mathrm{ZrO}_{7}$ particles and Gd123 phase also occurs in these doped bulks and produces $\mathrm{BaZrO}_{3}$ particles, accompanied by a certain amount of liquid phase compound. In addition, it can be found that the molar ratio of $\mathrm{Gd}$ to $\mathrm{Ba}$ in

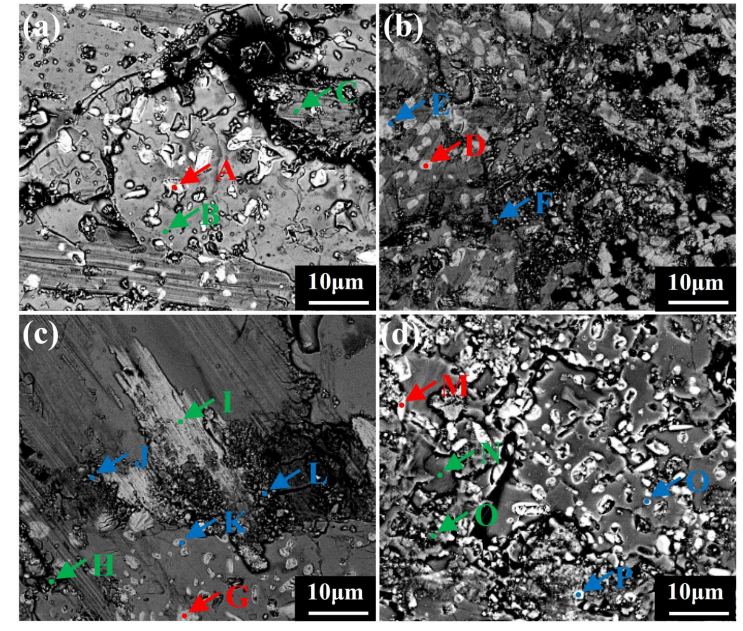

Fig. 10. SEM micrographs (at a magnification of $5000 \times)$ of specimens at B1 position of the GdBCO superconductor bulks with different doping amounts of $\mathrm{Gd}_{3} \mathrm{ZrO}_{7}$ particles: (a) S0, (b) S2, (c) S4, (d) S8. The Gd211 particles, Gd123 phase and spot containing $\mathrm{Gd} 211$ particles, $\mathrm{BaZrO}_{3}$ particles and liquid phase compound $\left(\mathrm{BaCuO}_{2}+\mathrm{CuO}\right)$ are marked with red dots, green dots, and blue dots, respectively.

the Gd123 phase of the doped bulks (spots H and I in $\mathrm{S} 4$ and spots $\mathrm{N}$ and $\mathrm{O}$ in $\mathrm{S} 8$ ) is higher than that of the undoped bulk (spots B and $\mathrm{C}$ in $\mathrm{S} 0$ ), that is, a more superconducting phase of doped bulks exists as the $\mathrm{Gd}_{1+x} \mathrm{Ba}_{2-x} \mathrm{Cu}_{3} \mathrm{O}_{7}$ phase. Considering the obvious second peak appeared in $\mathrm{S} 2$ and $\mathrm{S} 4$, we infer that the $\mathrm{Gd}_{3} \mathrm{ZrO}_{7}$ additions can intensify the $\mathrm{Gd} / \mathrm{Ba}$ substitution in the GdBCO bulks. One can suggest that the following reactions may occur during the growth of GdBCO superconductor bulks:

$$
\begin{aligned}
& \mathrm{GdBa}_{2} \mathrm{Cu}_{3} \mathrm{O}_{7}+\mathrm{Gd}_{3} \mathrm{ZrO}_{7} \rightarrow \\
& \mathrm{BaZrO}_{3}+4 \mathrm{Gd}^{3+}+\mathrm{BaCuO}_{2}+2 \mathrm{CuO} \\
& \mathrm{Gd}^{3+}+\mathrm{GdBa}_{2} \mathrm{Cu}_{3} \mathrm{O}_{7} \rightarrow \mathrm{Gd}_{1+x} \mathrm{Ba}_{2-x} \mathrm{Cu}_{3} \mathrm{O}_{7} \\
& 2 \mathrm{GdBa}_{2} \mathrm{Cu}_{3} \mathrm{O}_{7} \rightleftharpoons \\
& \quad \mathrm{Gd}_{2} \mathrm{BaCuO}+3 \mathrm{BaCuO}_{2}+2 \mathrm{CuO}
\end{aligned}
$$

Throughout the TSMG process, some part of the Gd123 phase reacts with $\mathrm{Gd}_{3} \mathrm{ZrO}_{7}$ particles generating $\mathrm{BaZrO}_{3}$ particles and releasing $\mathrm{Gd}^{3+}$ ions, as shown with (1). At high temperature, some other part of the Gd123 phase decomposes and generates Gd211 particles, as shown with (3).

As shown in (2), the released $\mathrm{Gd}^{3+}$ ions can substitute for the $\mathrm{Ba}^{2+}$ ions in the crystal lattice of $\mathrm{GdBa}_{2} \mathrm{Cu}_{3} \mathrm{O}_{7}$ and form the $\mathrm{Gd}_{1+x} \mathrm{Ba}_{2-x} \mathrm{Cu}_{3} \mathrm{O}_{7}$ phase. The $\mathrm{BaZrO}_{3}$ particles were widely used as the doping particles in many studies and proved to have a significant effect on the improvement of the superconducting property of the REBCO superconductors [15, 34-38]. In addition, some studies proposed that the $\mathrm{BaZrO}_{3}$ particles, which formed in the $\mathrm{ZrO}_{2}$-doped Gd123 bulk can disperse in the bulk 
Stoichiometry and possible particles of spots labeled in Fig. 10 identified by EDS analysis.

TABLE I

\begin{tabular}{|c|c|c|c|c|c|c|}
\hline Sample & Spot & $\operatorname{Gd}[\%]$ & $\mathrm{Ba}[\%]$ & $\mathrm{Cu}[\%]$ & $\mathrm{Zr}[\%]$ & Possible particles \\
\hline \multirow{3}{*}{ S0 } & $\mathrm{A}$ & 19.2 & 10.5 & 10.9 & 0.0 & Gd211 \\
\hline & $\mathrm{B}$ & 7.9 & 16.1 & 24.5 & 0.0 & $\operatorname{Gd} 123(\mathrm{Gd}: \mathrm{Ba}: \mathrm{Cu}=1: 2.04: 3.10)$ \\
\hline & $\mathrm{C}$ & 7.0 & 14.6 & 21.8 & 0.0 & Gd123 (Gd:Ba:Cu = 1:2.09:3.11) \\
\hline \multirow{3}{*}{$\mathrm{S} 2$} & $\mathrm{D}$ & 21.9 & 12.0 & 12.6 & 0.0 & Gd211 \\
\hline & $\mathrm{E}$ & 10.9 & 15.0 & 11.7 & 7.0 & $109 \mathrm{Gd} 211+140 \mathrm{BaZrO}_{3}+51 \mathrm{BaCuO}_{2}+74 \mathrm{CuO}$ \\
\hline & $\mathrm{F}$ & 1.5 & 15.3 & 5.1 & 9.8 & $15 \mathrm{Gd} 211+196 \mathrm{BaZrO}_{3}+95 \mathrm{BaCuO}_{2}$ \\
\hline \multirow{6}{*}{$\mathrm{S} 4$} & G & 18.4 & 9.9 & 10.3 & 0.1 & Gd211 \\
\hline & $\mathrm{H}$ & 7.7 & 15.1 & 22.4 & 0.0 & $\mathrm{Gd} 123(\mathrm{Gd}: \mathrm{Ba}: \mathrm{Cu}=1: 1.96: 2.91)$ \\
\hline & $\mathrm{I}$ & 3.8 & 7.2 & 11.8 & 0.0 & $\mathrm{Gd} 123(\mathrm{Gd}: \mathrm{Ba}: \mathrm{Cu}=1: 1.89: 3.11)$ \\
\hline & $\mathrm{J}$ & 5.3 & 15.7 & 16.2 & 4.5 & $53 \mathrm{Gd} 211+90 \mathrm{BaZrO}_{3}+171 \mathrm{BaCuO}_{2}+100 \mathrm{CuO}$ \\
\hline & $\mathrm{K}$ & 15.0 & 10.7 & 13.0 & 0.4 & $75 \mathrm{Gd} 211+4 \mathrm{BaZrO}_{3}+28 \mathrm{BaCuO}_{2}+27 \mathrm{CuO}$ \\
\hline & $\mathrm{L}$ & 8.4 & 9.2 & 8.5 & 1.1 & $42 \mathrm{Gd} 211+11 \mathrm{BaZrO}_{3}+39 \mathrm{BaCuO}_{2}+4 \mathrm{CuO}$ \\
\hline \multirow{5}{*}{$\mathrm{S} 8$} & M & 19.0 & 10.4 & 10.6 & 0.0 & Gd211 \\
\hline & $\mathrm{N}$ & 8.8 & 17.2 & 25.4 & 0.0 & Gd123 (Gd:Ba:Cu = 1:1.95:2.89) \\
\hline & $\mathrm{O}$ & 8.2 & 15.1 & 22.7 & 0.0 & Gd123 (Gd:Ba:Cu = 1:1.84:2.77) \\
\hline & $\mathrm{P}$ & 10.5 & 15.4 & 12.0 & 7.8 & $105 \mathrm{Gd} 211+156 \mathrm{BaZrO}_{3}+47 \mathrm{BaCuO}_{2}+88 \mathrm{CuO}$ \\
\hline & $\mathrm{Q}$ & 9.7 & 15.3 & 14.7 & 5.7 & $97 \mathrm{Gd} 211+114 \mathrm{BaZrO}_{3}+95 \mathrm{BaCuO}_{2}+102 \mathrm{CuO}$ \\
\hline
\end{tabular}

with the size of around $50 \mathrm{~nm}[34,35]$ — the size small enough to treat the $\mathrm{BaZrO}_{3}$ particles as the effective pinning centers. Therefore, as compared with the undoped bulk in which only Gd211 particles serve as the pinning centers, both $\mathrm{BaZrO}_{3}$ particles and Gd211 particles — as the pinning centers in the doped bulks - can well improve the flux pinning performance of GdBCO superconductor bulks.

When the GdBCO bulk is doped with the $\mathrm{Gd}_{3} \mathrm{ZrO}_{7}$ particles, some part of the Gd123 phase reacts with $\mathrm{Gd}_{3} \mathrm{ZrO}_{7}$ particles and, since such a Gd123 phase decomposition occurs, only a few of the Gd211 particles remain. Moreover, the liquid phase compound produced by the reaction between $\mathrm{Gd}_{3} \mathrm{ZrO}_{7}$ particles and the Gd123 phase can continually react with the Gd211 particles to promote the growth of the Gd123 superconducting phase, which can also decrease the content of the Gd211 particles. During the TSMG process, the Gd211 particles generally accumulate under the seed and are pushed to the growth front according to the pushing/trapping theory $[21,25,26]$. However, the decrease of the content of Gd211 particles can weaken the pushing effect, leading to fewer Gd211 particles to be pushed to the boundary.

This can well explain the phenomenon that the content of the Gd211 particles at the B1 position decreases with the increase of $\mathrm{Gd}_{3} \mathrm{ZrO}_{7}$ additions, as shown in Fig. 8. The $\mathrm{BaZrO}_{3}$ particles formed by the reaction between $\mathrm{Gd}_{3} \mathrm{ZrO}_{7}$ particles and $\mathrm{Gd} 123$ phase tend to accumulate at the early stage of crystal growth, that is, at the region under the seed. They diffuse to the boundary during the crystal growth. When the doping amount of the $\mathrm{Gd}_{3} \mathrm{ZrO}_{7}$ particles is low, the content of the $\mathrm{BaZrO}_{3}$ particles is low, thus the $\mathrm{BaZrO}_{3}$ particles are mainly distributed under the seed. As a result, the content of the $\mathrm{BaZrO}_{3}$ particles under the seed is higher than that near the boundary. When the doping amount of the $\mathrm{Gd}_{3} \mathrm{ZrO}_{7}$ particles is higher, the content of the $\mathrm{BaZrO}_{3}$ particles under the seed increases, and continuously diffuses to the boundary during the TSMG process.

Hence, we impose a view that for the low doping amount of the $\mathrm{Gd}_{3} \mathrm{ZrO}_{7}$ particles, the increase of the self-field $J_{c}$ at the position under the seed is due to the fact that more $\mathrm{BaZrO}_{3}$ particles accumulate under the seed and thus serve as the pinning centers (although the content of Gd211 particles under the seed decreases). However, for the high doping amount of the $\mathrm{Gd}_{3} \mathrm{ZrO}_{7}$ particles, less accumulation of the $\mathrm{BaZrO}_{3}$ particles greatly degrades the self-field $J_{\text {c }}$ property under the seed. It is due to the $\mathrm{Gd}_{3} \mathrm{ZrO}_{7}$ diffusion and the significant decrease of the content of Gd211 particles under the seed. It may be the reason why the self-field $J_{\mathrm{c}}$ of the specimens under the seed increases first, but insubstantially and even decreases significantly at the $\mathrm{C} 1$ position when the $\mathrm{Gd}_{3} \mathrm{ZrO}_{7}$ additions reach $0.8 \mathrm{~mol} \%$, as shown in Fig. 6. While in the region near the boundary, when the doping amount of the $\mathrm{Gd}_{3} \mathrm{ZrO}_{7}$ particles is low, the $\mathrm{BaZrO}_{3}$ particles mainly accumulate under the seed, thus there are few $\mathrm{BaZrO}_{3}$ particles as pinning centers near the boundary.

Additionally, the decrease of the content of Gd211 particles pushed to the boundary can also degrade the $J_{\mathrm{c}}$ properties of the specimens near the boundary. Therefore, the self-field $J_{\mathrm{c}}$ of the specimens near the boundary in S2 decreases, as compared with the undoped bulk. As the $\mathrm{Gd}_{3} \mathrm{ZrO}_{7}$ additions increase, more $\mathrm{BaZrO}_{3}$ particles are formed and serve as the flux pinning centers. Further, such $\mathrm{BaZrO}_{3}$ particles can diffuse to the boundary during 


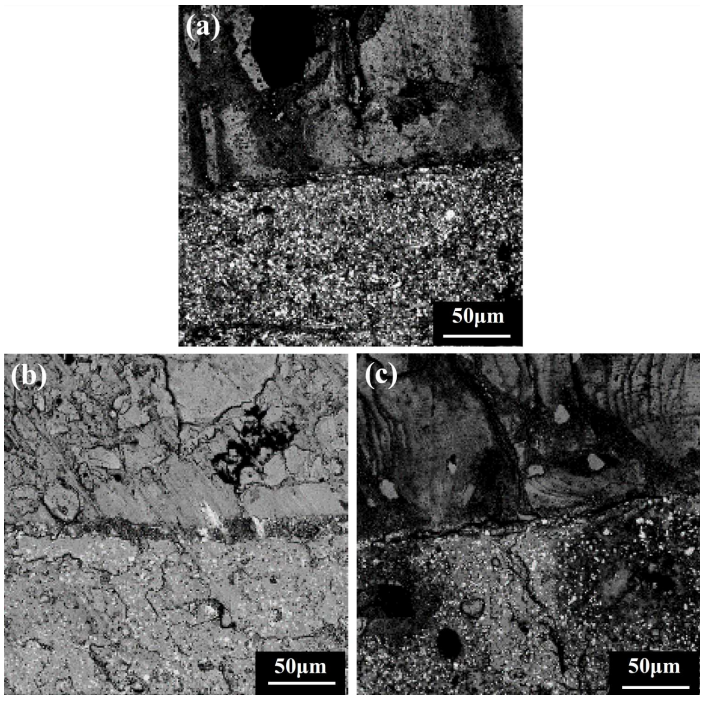

Fig. 11. SEM micrographs (at a magnification of $1000 \times)$ of the peculiar microstructure in doped GdBCO superconductor bulks with different amounts of $\mathrm{Gd}_{3} \mathrm{ZrO}_{7}$ particles: (a) $\mathrm{S} 2$, (b) S4, (c) S8.

the crystal growth, which results in higher self-field $J_{\text {c }}$ values of the specimens near the boundary with the addition of the $\mathrm{Gd}_{3} \mathrm{ZrO}_{7}$ particles. It is the reason why the self-field $J_{\text {c }}$ of the specimens near the boundary decreases first, then increases with the increase of $\mathrm{Gd}_{3} \mathrm{ZrO}_{7}$ additions, as shown in Fig. 6 . Since the amount of the $\mathrm{BaZrO}_{3}$ particles is high in B1 of S8, the critical radius of the Gd211 particle increases [22], that is, some Gd211 particles with a slightly larger size trapped in the GdBCO bulk doped with a smaller amount of the $\mathrm{Gd}_{3} \mathrm{ZrO}_{7}$ additions can be pushed to the boundary in S8, thus enhancing the average size of the Gd211 particles in B1 of S8. It may be the reason why the average size of Gd211 particles in S8 is higher than that of Gd211 particles in S2 and S4, as shown in Fig. 9.

In general, the $J_{\mathrm{c}}$ of REBCO bulks in low fields depends more on the $\delta l$ pinning, while the $J_{\mathrm{c}}$ of REBCO bulks in intermediate and high fields depends more on the $\delta T_{\mathrm{c}}$ pinning. In this study, the addition of $\mathrm{Gd}_{3} \mathrm{ZrO}_{7}$ particles provides more $\mathrm{BaZrO}_{3}$ particles as the pinning centers and intensifies the $\mathrm{Gd} / \mathrm{Ba}$ substitution. The former can lead to the $\delta l$ pinning centers which increase $J_{\mathrm{c}}$ in low fields, while the latter can result in the $\delta T_{\mathrm{c}}$ pinning centers which can increase $J_{\mathrm{c}}$ in intermediate and high fields and can be reflected by the second peak effect $[5,39]$. In fact, they cause the improvement of the $J_{\text {c }}$ property by doping $\mathrm{Gd}_{3} \mathrm{ZrO}_{7}$ particles, and the continuous increase of the trapped flux density with the increase of $\mathrm{Gd}_{3} \mathrm{ZrO}_{7}$ additions.

It can also be observed in Fig. 10 that the microstructure of the B1 of S4 exhibits obvious inhomogeneity. However, this peculiar microstructure is not unique to $\mathrm{S} 4$. Also, we can find such microstructural inhomogeneity in S2 and S8, as shown

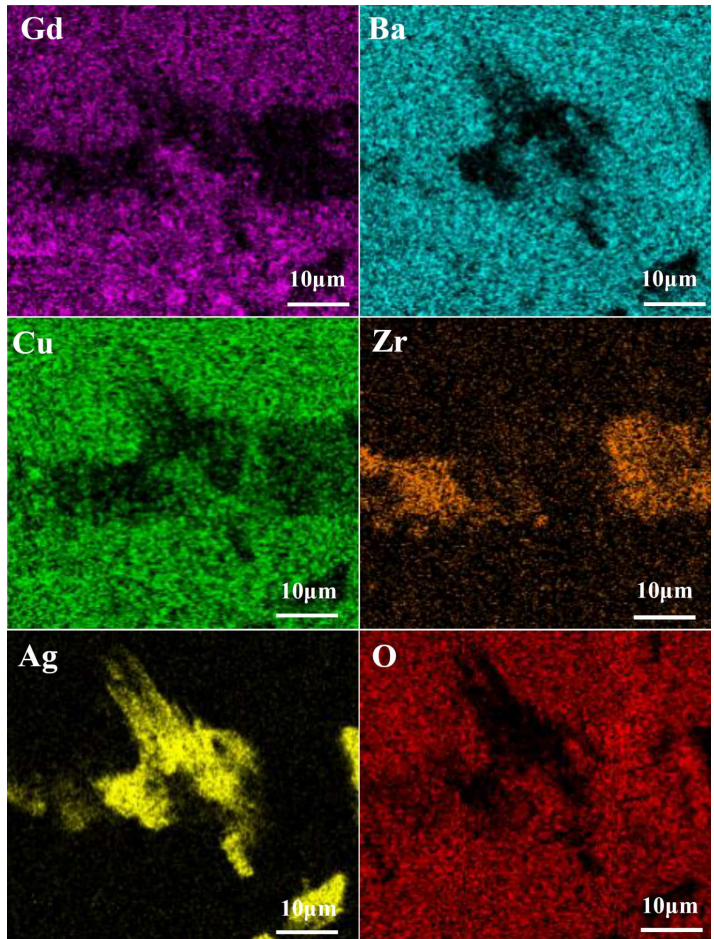

Fig. 12. EDS elemental maps of B1 specimen in S4 corresponding to Fig. 10c.

in Fig. 11 which gives SEM micrographs of doped GdBCO bulks at a lower magnification of $1000 \times$. Figure $11 \mathrm{~b}$ is a SEM diagram of the B1 specimen in S4 shown in Fig. 10c at a lower magnification. Figure 12 shows EDS elemental maps of the B1 specimen in $\mathrm{S} 4$ corresponding to Fig. 10c. It can be found that all doped samples form the microstructure with the Gd211-free region and the Gd211containing region separated by a special layer. The composition of this special layer was analyzed by EDS analysis (see Fig. 12). By combining EDS elemental maps in Fig. 12 and stoichiometry of spots $\mathrm{J}$ and $\mathrm{L}$ in this special layer shown in Fig. 10 and Table I, we can draw the following conclusion: the special layer, which separates the Gd211 free region and the Gd211-containing region is mainly made of $\mathrm{BaZrO}_{3}$ particles and $\mathrm{Ag}$ particles. Some studies on the REBCO bulks doped with Zr-containing particles have already proposed the idea of inhomogeneous microstructure $[18,21]$ and the phenomenon that Zr-containing particles tend to accumulate around silver [14]. Therefore, we also infer that during the TSMG process, $\mathrm{BaZrO}_{3}$ particles formed by a reaction between $\mathrm{Gd}_{3} \mathrm{ZrO}_{7}$ particles and the Gd123 phase can accumulate along with $\mathrm{Ag}$ and form the layer. This, in fact, can result in the formation of an inhomogeneous microstructure in which the Gd211 free region and the Gd211containing region were separated by the $\mathrm{BaZrO}_{3}$ and-Ag layer in a small region. Such an inhomogeneous microstructure results in the appearance of squared stripes around the seed crystal in the doped GdBCO superconductor bulks. According to Fig. 3, 
the region where the squared stripes are distributed becomes smaller with the increase of $\mathrm{Gd}_{3} \mathrm{ZrO}_{7}$ additions. It means that the microstructural uniformity can be improved with increasing $\mathrm{Gd}_{3} \mathrm{ZrO}_{7}$ additions, which is consistent with the previous analysis.

Some studies on the REBCO bulks doped with Zr-containing particles have already proposed the idea of inhomogeneous microstructure $[18,21]$ and the phenomenon that $\mathrm{Zr}$-containing particles tend to accumulate around silver [14].

\section{Conclusion}

In this study, we have successfully prepared four $\mathrm{GdBa}_{2} \mathrm{Cu}_{3} \mathrm{O}_{7-\delta}$ (GdBCO) single domain superconductor bulks doped with $0-0.8$ mol.\% $\mathrm{Gd}_{3} \mathrm{ZrO}_{7}$ particles by the modified TSMG method. The $\mathrm{Gd}_{3} \mathrm{ZrO}_{7}$ powders were prepared by solid state reaction and the single phase and high purity were identified by XRD analysis. The superconducting properties, such as critical temperature $\left(T_{\mathrm{c}}\right)$, critical current density $\left(J_{\mathrm{c}}\right)$ and trapped flux density $\left(B_{\text {trap }}\right)$, as well as the microstructure of these GdBCO bulks have been systematically analyzed. The onset critical temperature $\left(T_{\mathrm{c} \text {,onset }}\right)$ of all GdBCO superconductor bulks are above $94 \mathrm{~K}$, meaning the successful preparation and superior superconductivity.

The reaction between $\mathrm{Gd}_{3} \mathrm{ZrO}_{7}$ particles and Gd123 phase occurs during the TSMG process, forming the $\mathrm{BaZrO}_{3}$ particles and intensifying the $\mathrm{Gd} / \mathrm{Ba}$ substitution which leads to the $\delta l$ pinning centers and $\delta T_{\text {c }}$ pinning centers, respectively. Such an improvement of flux pinning performance significantly enhances $J_{\mathrm{c}}$ and the trapped flux density. The highest self-field $J_{\mathrm{c}}$ of $49.1 \mathrm{kA} / \mathrm{cm}^{2}$ was obtained in the GdBCO bulk doped with 0.8 mol.\% $\mathrm{Gd}_{3} \mathrm{ZrO}_{7}$ particles. Along with doping, the selffield $J_{\mathrm{c}}$ of the specimens under the seed first increases and then, in the case of the $\mathrm{C} 1$ position, it significantly decreases when the doping amount of $\mathrm{Gd}_{3} \mathrm{ZrO}_{7}$ particles reaches 0.8 mol. $\%$ while the self-field $J_{\mathrm{c}}$ of the specimens near the boundary decreases first, and then increases with the increase of the $\mathrm{Gd}_{3} \mathrm{ZrO}_{7}$ additions. It is due to the combined effect of the $\mathrm{Gd}_{2} \mathrm{BaCuO}_{5}(\mathrm{Gd} 211)$ particles and the $\mathrm{BaZrO}_{3}$ particles according to the SEM and EDS analysis. The trapped flux density of GdBCO bulks continuously increases with the doping amount of $\mathrm{Gd}_{3} \mathrm{ZrO}_{7}$ particles, and the maximum trapped flux density of $0.56 \mathrm{~T}$ is obtained in the bulk doped with $0.8 \mathrm{~mol}_{0} \% \mathrm{Gd}_{3} \mathrm{ZrO}_{7}$ particles, which is more than three times as high as that of the undoped bulk whose maximum flux density is $0.17 \mathrm{~T}$.

The doped bulks exhibit squared stripes around the seed crystal, which are identified as the macroscopic appearance of the inhomogeneous microstructure in which the $\mathrm{Gd}_{2} \mathrm{BaCuO}_{5}(\mathrm{Gd} 211)$ free region and the Gd211-containing region were separated by the $\mathrm{BaZrO}_{3}-$ and- $\mathrm{Ag}$ layer in a small region. The region where squared stripes are distributed becomes smaller with the increase of
$\mathrm{Gd}_{3} \mathrm{ZrO}_{7}$ particles, which means the microstructural inhomogeneity can be improved by the addition of the $\mathrm{Gd}_{3} \mathrm{ZrO}_{7}$ particles. These results confirm that the superconducting properties and microstructural uniformity of the GdBCO superconductor bulk can be well improved by doping $\mathrm{Gd}_{3} \mathrm{ZrO}_{7}$ particles, which is of significance for the industrial applications of superconductors.

\section{Acknowledgments}

This work was supported by the National Natural Science Foundation of China (grant No. 11004129), the Scientific Research Starting Foundation for the Returned Overseas Chinese Scholars, the Ministry of Education of China (SRF for ROCS, SEM), the Innovation Program of Shanghai Municipal Education Commission, China (grant No. 11YZ197, 12ZZ174), and the Shanghai Natural Science Foundation, China (grant No. 16ZR1422700).

\section{References}

[1] T. Ida, Z. Li, D.F. Zhou, M. Miki, Y.F. Zhang, M. Izumi, Supercond. Sci. Technol. 29, 054005 (2016).

[2] J. Wang, S. Wang, Y. Zeng et al., Physica C 378-381, 809 (2002).

[3] N. Koshizuka, Physica C 445-448, 1103 (2006).

[4] P. Mukherjee, V.V. Rao, Physica C 563, 67 (2019).

[5] Y.F. Zhang, D.F. Zhou, T. Ida, M. Miki, M. Izumi, Supercond. Sci. Technol. 29, 044005 (2016).

[6] K. Zmorayova, L. Vojtkova, T. Hlasek, J. Plechacek, P. Diko, Supercond. Sci. Technol. 33, 034005 (2020).

[7] W.M. Yang, X.D. Guo, F. Wan, G.Z. Li, Cryst. Growth Des. 11, 3056 (2011).

[8] Z.L. Feng, W.M. Yang, J.W. Li, M. Wang, Y.X. Yang, Z.B. Gao, X.N. Huang, J. Supercond. Nov. Magn. 33, 1559 (2020).

[9] L. Vojtkova, P. Diko, S. Piovarči, Acta Phys. Pol. A 131, 1048 (2017).

[10] S.P.K. Naik, M. Muralidhar, M. Murakami, J. Supercond. Nov. Magn. 31, 981 (2018).

[11] P. Hajdova, I. Shepa, E. Mudra, M. Rajnak, J. Dusza, P. Diko, Acta Phys. Pol. A 137, 800 (2020).

[12] D. Volochová, P. Diko, S. Piovarči, V. Antal, J. Kováč, M. Jirsa, Acta Phys. Pol. A 131, 1009 (2017).

[13] Y.F. Zhang, M. Izumi, Y.J. Li, M. Murakami, T. Gao, Y.S. Liu, P.L. Li, Physica C 471, 840 (2011). 
[14] D.F. Zhou, M. Izumi, T. Fujimoto, Y.F. Zhang, W.L. Zhou, K. Xu, IEEE Trans. Appl. Supercond. 25, 6800204 (2015).

[15] F. Wang, H. Tian, J. Mater. Sci. Mater. Electron. 30, 4137 (2019).

[16] C. Xu, A. Hu, N. Sakai, M. Izumi, I. Hirabayashi, Supercond. Sci. Technol. 18, 1082 (2005).

[17] K. Xu, D.F. Zhou, B.Z. Li, S. Hara, Z.G. Deng, M. Izumi, Physica C 510, 54 (2015).

[18] C. Xu, A. Hu, N. Sakai, M. Izumi, I. Hirabayashi, Physica C 445-448, 357 (2006).

[19] Y. Zhang, M. Izumi, Y. Kimura, Y. Xu, Physica C 469, 1169 (2009).

[20] D.X. Chen, R.B. Goldfarb, J. Appl. Phys. 66, 2489 (1989).

[21] K. Iida, N.H. Babu, E.S. Reddy, Y.H. Shi, D.A. Cardwell, Supercond. Sci. Technol. 18, 249 (2004).

[22] A.E. Carrillo, T. Puig, J. Plain, J. Figueras, X. Obradors, Physica C 336, 213 (2000).

[23] V. Antal, K. Zmorayova, M. Rajnak, L. Vojtkova, T. Hlasek, J. Plechacek, P. Diko, Supercond. Sci. Technol. 33, 044004 (2020).

[24] P. Hajdova, K. Zmorayova, M. Rajnak, P. Diko, Supercond. Sci. Technol. 33, 034003 (2020).

[25] Y.H. Shi, D.K. Namburi, W. Zhao, J.H. Durrell, A.R. Dennis, D.A. Cardwell, Supercond. Sci. Technol. 29, 015010 (2016).

[26] W. Zhai, Y.H. Shi, J.H. Durrell, A.R. Dennis, D.A. Cardwell, Cryst. Growth Des. 14, 6367 (2014).
[27] D.A. Cardwell, Y.H. Shi, N.H. Babu, S.K. Pathak, A.R. Dennis, K. Iida, Supercond. Sci. Technol. 23, 034008 (2010).

[28] S. Nariki, S.J. Seo, N. Sakai, M. Murakami, Supercond. Sci. Technol. 13, 778 (2000).

[29] D. Zhou, S. Hara, B. Li, J. Noudem, M. Izumi, Supercond. Sci. Technol. 27, 044015 (2014).

[30] D. Volochová, K. Jurek, M. Radušovská, S. Piovarči, V. Antal, J. Kováč, M. Jirsa, P. Diko, Acta Phys. Pol. A 126, 358 (2014).

[31] C.P. Bean, Rev. Mod. Phys. 36, 31 (1964).

[32] H.P. Liao, J. Zheng, L.W. Jin, H. Huang, Z.G. Deng, Y.H. Shi, D.F. Zhou, D.A. Cardwell, Supercond. Sci. Technol. 31, 035010 (2018).

[33] W.M. Yang, X.C. Yuan, C.Y. Zhang, IEEE Trans. Appl. Supercond. 29, 6802505 (2019).

[34] K.D. Keukeleere, P. Cayado, A. Meledin et al., Adv. Electron. Mater. 2, 1600161 (2016).

[35] A. Hu, C. Xu, M. Izumi, I. Hirabayashi, M. Ichihara, Appl. Phys. Lett. 89, 192508 (2006).

[36] C. Xu, A. Hu, M. Ichihara, M. Izumi, Y. Xu, N. Sakai, I. Hirabayashi, Jpn. J. Appl. Phys. 48, 023002 (2009).

[37] S. Kang, A. Goyal, J. Li et al., Science 311, 1911 (2006).

[38] A. Xu, Y. Zhang, M.H. Gharahcheshmeh et al., Sci. Rep. 7, 6853 (2017).

[39] M.R. Koblischka, A.J.J. van Dalen, T. Higuchi, S.I. Yoo, M. Murakami, Phys. Rev. B 58, 2863 (1998). 